Dynamics of converging laser-created plasmas in semi-cylindrical cavities studied using soft $x$-ray laser interferometry

M. A. Purvis, J. Grava, J. Filevich, M. Marconi, J. Dunn, S. J. Moon, V. N. Shlyaptsev, E. Jankowska, J. J. Rocca

September 26, 2007

Physical Review E 
This document was prepared as an account of work sponsored by an agency of the United States Government. Neither the United States Government nor the University of California nor any of their employees, makes any warranty, express or implied, or assumes any legal liability or responsibility for the accuracy, completeness, or usefulness of any information, apparatus, product, or process disclosed, or represents that its use would not infringe privately owned rights. Reference herein to any specific commercial product, process, or service by trade name, trademark, manufacturer, or otherwise, does not necessarily constitute or imply its endorsement, recommendation, or favoring by the United States Government or the University of California. The views and opinions of authors expressed herein do not necessarily state or reflect those of the United States Government or the University of California, and shall not be used for advertising or product endorsement purposes. 


\title{
Dynamics of converging laser-created plasmas in semi-cylindrical cavities studied using soft $x$-ray laser interferometry
}

\author{
Mike Purvis ${ }^{1}$, Jonathan Grava ${ }^{1}$, Jorge Filevich ${ }^{2}$, Mario C. Marconi ${ }^{1}$, James Dunn ${ }^{3}$, \\ Stephen J. Moon ${ }^{3}$, Vyacheslav N. Shlyaptsev ${ }^{4}$, Elizabeth Jankowska ${ }^{5}$, and Jorge J. \\ Rocca $^{1,2}$ \\ ${ }^{1}$ NSF ERC for Extreme Ultraviolet Science and Technology and Department of Electrical and Computer Engineering, \\ Colorado State University, Fort Collins, Colorado 80523, USA \\ ${ }^{2}$ NSF ERC for Extreme Ultraviolet Science and Technology and Department of Physics, Colorado State University, \\ Fort Collins, Colorado 80523, USA \\ ${ }^{3}$ Lawrence Livermore National Laboratory, Livermore, California 94551, USA \\ ${ }^{4}$ Department of Applied Science, University of California Davis-Livermore, Livermore, California 94551, USA \\ ${ }^{5}$ Wroclaw University of Technology, Wroclaw, Poland
}

PACS number(s): 52.50.Jm, 52.65.-y, 52.70.-m, 42.55.Vc

\section{Abstract}

The evolution of dense aluminum and carbon plasmas produced by laser irradiation of $500 \mu \mathrm{m}$ diameter semi-cylindrical targets was studied using soft x-ray laser interferometry. Plasmas created heating the cavity walls with 120 picosecond duration optical laser pulses of $\sim 1 \times 10^{12} \mathrm{~W}$ $\mathrm{cm}^{-2}$ peak intensity were observed to expand and converge on axis to form a localized high density plasma region. Electron density maps were measured using a $46.9 \mathrm{~nm}$ wavelength tabletop capillary discharge soft $\mathrm{x}$-ray laser probe in combination with an amplitude division interferometer based on diffraction gratings. The measurements show that the plasma density on axis exceeds $1 \times 10^{20} \mathrm{~cm}^{-3}$. The electron density profiles are compared with simulations conducted using the hydrodynamic code HYDRA, which show that the abrupt density increase near the axis is dominantly caused by the convergence of plasma generated at the bottom of the groove during laser irradiation. 


\section{Introduction}

The study of the dynamics of dense plasmas created by laser irradiation of cylindrical cavities is of interest for both fundamental plasma physics understanding and applications. The latter includes the hohlraums used in the implementation of indirect drive inertial confinement fusion. Laser heating of cylindrical hohlraums $[1,2]$ creates pressure gradients near the walls that radially accelerate the plasma distributed along the surface towards the axis of the cavity, where it collides. This interaction of converging plasmas depends on their degree of collisionality and can range from stagnation to extended interpenetration, as determined by the geometry and irradiation conditions [3]. Comparison of direct measurements of the electron density evolution with code simulations can contribute to a better understanding of the plasma dynamics, serve to benchmark these complex codes and determine their regimes of validity.

Imaging techniques, spectroscopy, and interferometry techniques have been used to study several different types of converging plasmas with different degrees of collisionality [4-8]. Bosch et al. used holographic interferometry at $\lambda=263 \mathrm{~nm}$ to obtain electron density maps of hot high density colliding plasmas [9]. However, complicated fringe patterns resulting from electron densities that exceed the limits of optical interferometry only allowed the electron density to be measured off axis, where the density is lower. The maximum plasma density and plasma size that can be probed can be increased using a shorter wavelength laser probe. Wan et al. used a soft xray laser probe at $\lambda=15.5 \mathrm{~nm}$ to perform an interferometry experiment of a colliding plasma created by irradiating two Au slabs aligned at $45^{\circ}$ with respect to the heating pulse axis, separated by a variable gap between the tips of the slabs. The walls of this "V" shape target were irradiated at intensities of $3 \times 10^{14} \mathrm{~W} \mathrm{~cm}^{-2}$ with a $1 \mathrm{~ns}$ long temporally square laser pulse [10]. The plasma collision at the plane of symmetry was measured to build up to a density of $6 \times 10^{20} \mathrm{~cm}^{-3}$ on axis. The low repetition rate at which the experiment could be performed allowed the acquisition of 
interferograms only at a few instances during the plasma evolution. Experimentally it was observed that as the plasma evolved the collisionality changed from early time interpenetration into later time stagnation. A comparison was made with the Lagrangian hydrodynamic code LASNEX. It was reported that the code predicted shock heated electron density peaks located off the symmetry plane, which were not observed in the experiment.

In this paper, we report results of an interferometry experiment designed to study the evolution of dense converging plasmas created by irradiation of a semi-cylindrical target at an intensity of $\sim 1 \times 10^{12} \mathrm{~W} \mathrm{~cm}^{-2}$ with laser pulses of $120 \mathrm{ps}$ duration. The plasmas were probed with the $46.9 \mathrm{~nm}$ beam from a compact capillary discharge soft x-ray laser (SXRL) [11] using a high throughput interferometer that uses diffraction gratings to split and recombine the beam [12]. The high repetition rate of the capillary discharge laser allowed us to obtain numerous interferograms that map the entire evolution of aluminum and carbon plasmas with high contrast soft $\mathrm{x}$-ray laser interferograms. The density maps obtained from these interferograms describe the radial expansion of the plasma towards the axis of the cavity, where a dramatic increase in the electron density is observed. Comparison of the data with 2-Dimensional (2D) simulations performed using the hydrodynamic code HYDRA, show very good agreement, and indicates that this behavior is dominantly caused by plasma converging on axis from a region located at the back of the target surface. This paper starts with a description of the experimental set up, followed by a summary of the interferometry results, and a comparison of the experimental measurements with simulations. The results describe the dynamics responsible for the formation of an electron density build-up resulting from converging plasmas expanding from semi-cylindrical cavities.

\section{Experimental setup}

The plasmas were created by irradiating half-cylinder cavities at intensities of $1 \times 10^{12} \mathrm{Wcm}^{-2}$ with $\lambda=800 \mathrm{~nm}$ pulses of $120 \mathrm{ps}$ (FWHM) duration from a Ti:Sapphire laser. The target geometry 
is schematically illustrated in Fig. 1. Sets of half-cylinder grooves $500 \mu \mathrm{m}$ in diameter were machined on the edge of $1 \mathrm{~mm}$ thick aluminium or carbon slabs with a spacing of $1 \mathrm{~mm}$ between grooves. A single slab comprised 35 accessible grooves, allowing numerous interferograms to be obtained without having to vent the vacuum target chamber. Two interferograms were obtained per groove. The target was mounted on a motorized $\mathrm{X}, \mathrm{Y}, \mathrm{Z}$ stage with rotation along the vertical axis that enabled precise positioning with respect to the SXRL probe beam. The optical laser beam irradiating the cavity impinged into it at normal incidence with respect to the flat front surface of the target and the SXRL probe beam propagated along the axis of the half-cylinder cavity.

A schematic of the entire experimental setup is shown in Fig. 2. The laser pulses used to create the plasma were produced by a Ti:Sapphire laser consisting of a Kerr mode-locked oscillator and three stages of amplification, yielding pulses of 120 ps duration. The present experiments used $600 \mathrm{~mJ}$ energy per pulse. The short pulse duration allows the rapid deposition of laser energy, making it possible to study the evolution of the plasma in the cavity without further laser heating of the plasma. A vacuum spatial filter was used to smooth the laser beam profile and improve the uniformity of the laser energy deposition onto the target. The pulse was focused by a $7 \mathrm{~m}$ focal length spherical lens to form a spot of $300 \mu \mathrm{m}$ FWHM diameter onto the target, which was stretched into a line by a pair of $1 \mathrm{~m}$ focal length positive and negative cylindrical lenses. In this experiment the cylindrical lens separation was set at $4.5 \mathrm{~cm}$ to create an effective focal length of $22.2 \mathrm{~m}$, which when combined with the astigmatism of the beam yielded a $1.5 \mathrm{~mm}$ FWHM long line focus on target. The homogeneity of the line focus was monitored on every shot by imaging it on an equivalent focal plane. This was performed by taking a reflection of the beam from a $4 \%$ beam splitter before it enters the vacuum system and by redirecting this reflection onto a CCD camera that was placed at the same distance from the beam splitter as the target. 
Interferograms of the plasma were obtained combining a table-top $46.9 \mathrm{~nm}$ capillary discharge laser [11] with an amplitude division soft x-ray interferometer developed at Colorado State University [12]. The interferometer is based on a skewed Mach-Zehnder geometry in which both the beam splitters and the refracting mirrors are placed at grazing incidence for increased reflectivity. Ruled diffraction gratings with gold coatings are used as beam splitters. The gratings contribute to the robustness of the design. The blaze angle of the gratings is selected to direct equal intensity of the beam into the zero and first order. The instrument has a throughput of $6 \%$ per arm. More details on the interferometer and its alignment procedure, which is performed with the assistance of an infrared laser diode, can be found in previous publications $[12,13]$. The target was placed along the zero order path of the interferometer between the grazing incidence gold mirror and the second diffraction grating. The timing of the arrival of the probe pulse with respect to the plasma heating pulse was measured using two photodiodes. The Ti:Sapphire laser pulse was detected by positioning a fast photodiode behind the last laser turning mirror, and the soft x-ray laser pulse was monitored with a vacuum photodiode placed inside the interferometer vacuum chamber to capture the higher orders of the first grating.

The probe beam was generated by a high repetition rate table-top $46.9 \mathrm{~nm}$ Ne-like Ar capillary discharge laser $[11,14]$ that delivered pulses of $\sim 1 \mathrm{~ns}$ duration and $\sim 0.15 \mathrm{~mJ}$ of energy. The good spatial coherence of this laser assists in the generation of interferograms with high fringe visibility [15]. The inherent jitter associated with the spark gap switch that governs the firing of the capillary discharge was reduced to $\sim 2$ ns by laser triggering it. This was achieved focusing 30 $\mathrm{mJ}$ pulses from a Nd:YAG laser onto one electrode of the spark gap where it created a plasma that initiated the breakdown of the switch. With this method of triggering it was possible to acquire a series of interferograms that describe the entire plasma evolution using a single target strip. 


\section{Plasma interferometry results}

A sequence of interferograms describing the evolution of aluminium plasmas created by irradiating the semi-cylindrical targets is shown in Fig. 3. Each interferogram corresponds to a different time measured with respect to the arrival of the $600 \mathrm{~mJ}$ heating pulse onto the target surface. The first frame, taken at $0.8 \mathrm{~ns}$, shows fringe shifts near the target surface that signal the creation of a plasma. The fringes at the center of the cavity are also slightly perturbed, indicating that a low density plasma is beginning to converge on axis. As early as $2.6 \mathrm{~ns}$ into the evolution, a large number of localized fringe shifts are observed just outside the geometrical axis of the curved target signifying the presence of a large electron density. In the frames of Fig. 3 corresponding to the times from 1.3 to $4.3 \mathrm{~ns}$, a small blurred region is observed near the axis that is discussed later and shown to be the result of rapid plasma motion. For delays of the order of 6 ns, absorption of the SXRL probe beam is beginning to be noticed near the walls due to the presence of a dense cold plasma. The absorption increases in time as the plasma continues to cool. This strong attenuation of the probe beam is caused by photoionization of low charge $\mathrm{Al}$ ions (ie. AlII, AlIII) and neutral atoms, resulting from plasma recombination, for which the photoionization potential is less than the $26.5 \mathrm{eV}$ energy of the photons in the probe beam. However, fringe visibility remains high farther away from the target and in the region where the density increase is observed. Furthermore, absorption does not preclude obtaining clear interference fringes at longer time delays such as $10.7 \mathrm{~ns}$.

Electron density maps constructed from each of the aluminium interferograms in the sequence described above are shown in Fig. 4. The data shows that the plasma expands away from the surface of the target, converging in a small region just outside the axis of the cavity causing an abrupt increase in the electron density. The increase in electron density develops as early as $1.3 \mathrm{~ns}$, reaching a measured maximum of $1.1 \times 10^{20} \mathrm{~cm}^{-3}$ at $2.6 \mathrm{~ns}$. The plasma in this 
region is observed to maintain the high electron density well after the time of its build up. A local density maximum of $7 \times 10^{19} \mathrm{~cm}^{-3}$ is still present as late as $10.7 \mathrm{~ns}$. This increase in plasma density is accompanied by a strong localized emission of radiation from that region, as shown in the timeintegrated self emission image of Fig. 5 which was taken using a micro channel plate sensitive to extreme ultraviolet and vacuum ultraviolet light.

A sequence of interferograms corresponding to carbon plasmas obtained by irradiation of semi-cylindrical targets, are shown in Fig. 6. These interferograms were taken under the same target irradiation conditions as those used in the aluminum experiment. The first interferogram of the sequence was acquired at the time of the peak of the 120 ps heating pulse. Plasma absorption and fringe shifts are present in the region within $\sim 50 \mu \mathrm{m}$ of the target surface, indicating the early stage of the plasma expansion. In the $1.3 \mathrm{~ns}$ frame perturbed fringes are observed nearly everywhere within the cavity. Near the center of the groove we observe a region, shaped like a horizontal "Y" in which it is not possible to resolve the fringes, for reasons later discussed. This frame indicates that at $1.3 \mathrm{~ns}$ the plasma has already converged on axis and a fast density build-up is under way near the axis of the cavity. At $2 \mathrm{~ns}$ after the irradiation significant shifts of the fringes are observed in an oval shaped region near the axis, indicating the formation of a strongly pinched plasma. At $2.6 \mathrm{~ns}$ the fringes in this location remain highly perturbed, and absorption of the probe beam is observed near the surface of the target. The subsequent frame, at $5.0 \mathrm{~ns}$, shows a significant decrease in the number of fringe shifts near the axis. The shifting continues to decrease with time until $\sim 10 \mathrm{~ns}$, at which the fringe visibility is lost due to significant absorption of the probe beam.

The electron density maps resulting from the carbon plasma interferograms are shown in Fig. 7. The early 1.3 ns frame reveals no density information within the center of the white "Y" shaped region. However, the peak electron density is observed to occur significantly earlier than for aluminium, and by $2.6 \mathrm{~ns}$ the density in this region has already begun to decrease. While from 
the measured fringe shifts it is possible to construct electron density maps, the assumption that only free electrons contribute to the refractive index is not valid for the colder regions of the carbon plasma. This reduces the region where accurate electron density maps can be obtained. In recent soft x-ray laser interferometry experiments we have observed anomalous fringe shifts caused by the contribution of bound electrons to the index of refraction[16]. For a probe laser wavelength of $46.9 \mathrm{~nm}$ anomalous fringe shifts were observed in tin, silver [17] and carbon [18] plasmas. In the case of the carbon plasmas it was found that the neutral atoms as well as singly and doubly ionized atoms can make significant contributions to the index of refraction. Therefore it is justifiable to assume that in regions where the mean degree of ionization is 3 or larger only the free electrons contribute to the index of refraction. In practice only the presence of doubly charged ions is of concern, as significant concentrations of neutral atoms or singly ionized ions greatly attenuates the probe beam by photoionization. We used simulations conducted with the code HYDRA discussed in the next section to determine the plasma regions in which the free electron approximation can be considered valid and consequently the electron density maps accurate. The region to the right of the thin contour lines in Figs. 6 and 7 correspond to the simulated plasmas with mean degree of ionization larger than 3. In these regions, that includes the zone of high density build-up, the approximation that only free electrons contribute to the index of refraction should be valid and therefore the electron density maps can be expected to be accurate. In addition, the region where the high density build-up was measured falls far from this boundary region and has a mean ionization of $Z \sim 5$.

\section{Simulations and discussion}

The converging plasmas were modeled with HYDRA, a three-dimensional (3D) single fluid radiation hydrodynamics code that utilizes a structured mesh made up of hexahedrons [19, 20]. We chose to set the dimension of a unit cell along the target axis to equal the plasma length, 
effectively creating a 2D mesh. The good uniformity of the line focus which overfills the target helps to justify the assumption of uniformity along this dimension.

Inverse Bremsstrahlung absorption is assumed to be the dominant laser deposition mechanism at our irradiation conditions. Radiation transport within the plasma was treated using multi-group diffusion techniques and tabulated opacities, and heat conduction was simulated using the conductivities of Lee and More [21]. The equations of state as well as the opacities are modeled using the Lawrence Livermore National Laboratory's LEOS library. The electron flux limiter was set to a value of 0.05 . Simulations performed on the carbon targets without radiation transport were not significantly different from those performed with radiation transport. However, the inclusion of radiation transport did improve the agreement between the simulated and measured plasma densities in the case of the aluminum plasmas. In the simulations with radiation transport the aluminum plasma reaches a peak in the electron density at $2.9 \mathrm{~ns}, \sim 500 \mathrm{ps}$ later than the simulations without radiation transport, in agreement with the experiment.

HYDRA is capable of running in Lagrangian mode, Eulerian mode or an Arbitrary Lagrangian Eulerian (ALE) hydrodynamic mode. An assortment of hydrodynamic mesh motion strategies, such as ALE, are available to help elude mesh tangling that commonly occurs when modeling a converging plasma. We chose a strategy in which the grid motion was set to be fully Lagrangian during the duration of the laser irradiation pulse. After the termination of the laser pulse we allowed the grid to move in the ALE mode. This strategy is well justified due to the fact that during the duration of the laser pulse the plasma blowoff does not reach the axial region, where severe mesh entanglement would occur. Therefore, the Lagrangian approach is most adequately used during this initial time interval. The ALE approach adaptively follows the expanding plasma and accurately reproduces shock waves automatically, the mesh is fine where density and its gradients are large and coarse when plasma rarifies. 
The simulated HYDRA electron density and electron temperature maps for the aluminium plasmas are presented in Figs. 8 and 9 respectively. The computed electron density maps are consistent with the measured electron density distributions in Fig. 4. The code reveals that the low density front of the expanding plasma is already beginning to interact near the axis of the target at $\sim 1$ ns. Simulations and experimental results show that the plasma density peak forms in a region near the groove's axis. As more plasma arrives to this region the density peak moves away from the surface of the target, reaching a maximum value in a region just off the axis of the groove. In the simulations the electron density reaches a peak value of $1.1 \times 10^{20} \mathrm{~cm}^{-3}, 35 \mu \mathrm{m}$ off the axis at 2.9 ns into the plasma evolution, in good agreement with the measurements. The electron density in this region is computed to remain high over the next few nanoseconds in agreement with the measurements which also show a high electron density well after the density build-up reaches a peak. This effect, unlike the focusing of light where photons do not interact with each other, is the result of increased repulsion in progressively denser plasma. Interpenetration occurs throughout the very early stages of the evolution when the density is small and ion speed is large. Moreover, interpenetration conditions exist during the onset of a density build-up, at a time when the plasma density is in the range of (1-3) $10^{19} \mathrm{~cm}^{-3}$ and the ion energy of the plasma edge expands with a speed of (1-3) $10^{7} \mathrm{~cm} / \mathrm{s}$, which corresponds to a kinetic energy of ions between 5 and $30 \mathrm{keV}$. For these conditions the size of the plasma focal spot is small and the ion-ion elastic collision mean free path is much larger than this focal spot. As the plasma density increases the plasma rapidly becomes highly collisional and a single fluid hydrodynamic code like HYDRA, which does not account for interpenetration, can accurately simulate the plasma. The code also reveals that the electron temperature at this location initially increases to a value of $\sim 60 \mathrm{eV}$ and subsequently is replaced by colder plasma that expands from the back of the target. When the electron density near the axis reaches its peak the electron temperature is computed to be $\sim 40 \mathrm{eV}$. 
HYDRA was also used to study the dynamics of carbon plasmas. Simulations for the carbon electron density and temperatures are shown in Fig. 10 and 11 respectively. Again, the computed electron density distributions compare well with the experimental data of Fig. 7 for the entire plasma region where the measurements are valid. As the simulated plasma expands, its low density plasma front begins to converge and collide as early as $800 \mathrm{ps}$ after the target irradiation, matching the measurements. This early plasma convergence strengthens significantly during the next nanosecond, forming a concentrated high density plasma near the axis of the cavity. This "plasma focus" reaches a peak electron density of $\sim 1 \times 10^{20} \mathrm{~cm}^{-3} \sim 25 \mu \mathrm{m}$ off the axis, $2.3 \mathrm{~ns}$ after laser irradiation. The code shows the electron temperature increasing to $\sim 45 \mathrm{eV}$ at $600 \mathrm{ps}$ and subsequently cooling to a value of $\sim 35 \mathrm{eV}$ at the time when the electron density peaks. The mean degree of ionization near the axis is computed to be $Z=5-5.5$ throughout the formation of the density build-up.

The simulated electron density maps also make it possible to explain the observed blurred horizontal "Y" shaped region appearing in the 1.3, 2.0 and $2.6 \mathrm{~ns}$ carbon interferograms shown in Fig. 6 . The local variations of the density during the $\sim 1$ ns duration of the probe pulse, which can lead to blurring of the fringes, can be determined by subtracting two computed electron density maps corresponding to that time difference. The simulations shown in Fig. 12 are maps of the difference in electron density over 1 ns periods centered at 1.3, 2.0 and 2.6 ns. Since one fringe shift at our probe wavelength corresponds to a density of $5 \times 10^{19} \mathrm{~cm}^{-3}$, the computed change in density of $2.5 \times 10^{19} \mathrm{~cm}^{-3}$ over $1 \mathrm{~ns}$ is large enough to blur the fringes. A " $\mathrm{Y}$ " shaped region can be seen in each of the frames of Fig. 12 that matches well the blurred region observed in the interferograms of Fig. 6. The shape and location of these regions are in agreement with the unresolved zone in the $1.3 \mathrm{~ns}$ interferogram in Fig. 6 . The fact that the intensity in the blurred region of the interferograms averages to $98 \%$ of the light at this location from a shot earlier in the 
time evolution where blurring is not observed, confirms that motion blurring and not refraction is the dominant cause for the loss of fringe visibility in this region.

The possible role of interpenetration within the plasmas that converge in the focal region can be determined by computing the ion-ion collision mean free path accounting for the relative drift between ions. Decoster and Jones [22] have performed an analysis for counter-streaming plasmas yielding two ion-ion collision frequencies, one for dynamic friction, and another related to temperature equilibration[22], determined by the ratio of the corresponding plasma velocity and collision mean free path. The semi-cylindrical surface of our target requires the consideration of an angle dependent drift velocity in which the counter streaming component of the drift velocity is minimized for small angles. Using the measured density data for the focal region and the simulated temperature and degree of ionization data from HYDRA the ion-ion collision mean free path for similar species can be computed to be less than $2.2 \mu \mathrm{m}$ which is small compared to the plasma dimensions. Further proof that interpenetration is not important at these conditions is the good agreement between the measured density maps and the results from the fluid code HYDRA which does not account for interpenetration.

The target irradiation intensity is maximum at the bottom of the groove, as a result of the combined effect of the nearly Gaussian profile of the laser that peaks at the center of the groove and the curvature of the target which presents a decreasing cross sectional area to the wings of the heating beam. Nearly $77 \%$ of the laser energy is deposited onto the target surface within the 74 degree angle segment corresponding to the heating beams FWHM as illustrated in Fig. 1. Therefore the plasma generated near the bottom of the groove is hotter and expands with a higher velocity, arriving to the focal region earlier. The expanding plasma generated in the upper and lower portions of the cavity is accelerated by significantly smaller pressure gradients and arrives on axis later in time. 
To better determine the relative contributions of the different regions of the wall to the plasma build-up in the focal region, tracer particle trajectories were computed. HYDRA was used to calculate vector fields at $10 \mathrm{ps}$ intervals which were then used to define the trajectory of a set of tracer particles that were intitially uniformly spaced along the curved surface of the target. Figure 13 shows the trajectory of particles ablated at times of $70,120,150$ and 200 ps with respect to the beginning of the heating pulse. Each frame follows the tracer particles from the time they leave the surface (the dark blue color) up to $4 \mathrm{~ns}$ in the evolution (the yellow color). Each alternating color illustrates the trajectories over an elapsed time of $500 \mathrm{ps}$. The first particles to leave the target surface expand into vacuum accelerated outward by the increasing pressure gradients. The converging plasma emanating from within the 74 degree central region of the target at the time of the peak of the irradiation ( $70 \mathrm{ps}$ frame) reaches the focus region in about $1.5 \mathrm{~ns}$, causing an abrupt increase in the plasma density. Plasma leaving this region of the target at the end of the pulse (120 ps frame) arrives to the focus region at 2 ns contributing significantly to the density build-up. In contrast, the tracer particles that start the evolution at target surface locations irradiated by a reduced intensity (larger angles) are noticeably slower and reach the focal region significantly later, near the end of the 4 ns evolution. Plasma radiation continues to ablate the target after the termination of the heating laser pulse. The trajectory plots that follow the particles which leave the target after the termination of the irradiation pulse (i.e. 150 and 200 ps frames) show this plasma moves significantly slower as a result of the colder temperature near the walls. Furthermore, plasma leaving the surface of the target at 200 ps does not reach the focal region where a plasma build-up occurs during the first $4 \mathrm{~ns}$ of the evolution. At this time all the tracer particles leave the target surface with similar velocity, independently of their position on the target surface. The density increase in the focal region is therefore dominantly caused by the convergence of plasma created at the bottom of the groove during the laser irradiation with additional contributions from plasma originating from the steeper wall of the cavity that arrives 
later and collides with little interpenetration. Fed mainly from the back of the target, the density increase occurs slightly off the axis of the cylinder due to the strong horizontal velocity component of this dominant plasma source. These trajectory plots also show a reduction in the vertical component of the expansion velocity, creating a narrowed stream of plasma that exits the cavity.

By following the tracer particles and computing its ionization throughout its trajectory we were able to determine a slight increase in ionization of $\sim 10 \%$ occurring while the plasma radially converges on axis. The increase in the ionization rate associated with the increased density in this region is compensated by the increase in collisional electron-ion recombination at the relatively small temperatures in this region.

\section{Conclusions}

We have studied the dynamics of plasmas generated by irradiation of semi-cylindrical aluminum and carbon cavities with 120 ps duration optical laser pulses at an intensity of $1 \times 10^{12}$ $\mathrm{W} \mathrm{cm}^{-2}$. Electron density maps obtained with the use of a soft $\mathrm{x}$-ray interferometer show that the plasma expands away from the target surface, converging slightly off axis, where a bright high density plasma focus with an electron density higher than $1 \times 10^{20} \mathrm{~cm}^{-3}$ is formed. The combination of the interferometry results with 2-dimensional simulations conducted with the hydrodynamic code HYDRA enabled to construct a picture of the expansion and evolution of the plasmas. The code simulations were also used to compute the degree of ionization of the plasma, which plays a role in determining the region of validity of the free electron approximation to the index of refraction of the plasma, and consequently the region of validity of the density maps obtained from the interferograms using this approximation.

Due to the uneven heating of the target a hotter plasma is created at the bottom of the groove. This hotter plasma expands faster reaching the axis of the groove first, where it converges and 
forms the dense plasma focus. This faster and hotter plasma is followed by the arrival of successively colder and slower plasma created by plasma radiation-induced ablation of the target after the termination of the laser pulse. The simulations show that the plasma collision in the focal region redirects the velocity narrowing the stream of plasma that leaves the cavity. Because the plasma is highly collisional, interpenetration is small, and the hydrodynamic code is capable of reliably computing the electron density distribution and resulting in a good agreement between the measured and simulated electron density maps. Collisions in the plasma focus region do not significantly increase the degree of ionization of the plasma. The plasma build-up occurring slightly off axis of the groove axis is instead mainly the result of the convergence of the plasma in a small region. The results also illustrate that the combination of soft x-ray laser interferometry with 2-dimensional hydrodynamic simulations is a powerful tool to study the dynamics of dense plasmas.

\section{Acknowledgements}

The authors would like to thank M. Marinak for the helpful discussions regarding the HYDRA simulations. This research was sponsored by the National Nuclear Security Administration under

the Stewardship Science Academic Alliances program through U.S. Department of Energy Research Grant \#DE-FG52-06NA26152, using facilities from the NSF ERC Center for Extreme Ultraviolet Science and Technology, award EEC-0310717. Part of this work was performed under the auspices of the U.S. Dept. of Energy by the University of California, Lawrence Livermore National Laboratory through the Institute of Laser Science and Application, under contract No. W-7405-Eng-48. 


\section{References}

[1] J. Lindl, Phys. Plasmas 2, 3933 (1995).

[2] T. R. Dittrich, S. W. Haan, M. M. Marinak, S. M. Pollaine, D. E. Hinkel, D. H. Munro, C. P. Verdon, G. L. Strobel, R. McEachern, R. C. Cook, C. C. Roberts, D. C. Wilson, P. A. Bradley, L. R. Foreman, and W. S. Varnum, Physics of Plasmas 6, 2164 (1999).

[3] M. E. Jones, D. Winske, S. R. Goldman, R. A. Kopp, V. G. Rogatchev, S. A. Bel'kov, P. D. Gasparyan, G. V. Dolgoleva, N. V. Zhidkov, N. V. Ivanov, Y. K. Kochubej, G. F. Nasyrov, V. A. Pavlovskii, V. V. Smirnov, and Y. A. Romanov, Phys. Plasmas 3, 1096 (1996).

[4] O. Rancu, P. Renaudin, C. Chenais-Popovics, H. Kawagoshi, J. C. Gauthier, M. Dirksmoller, T. Missalla, I. Uschmann, E. Forster, O. Larroche, O. Peyrusse, O. Renner, E. Krousky, H. Pepin, and T. Shepard, Physical Review Letters 75, 3854 (1995).

[5] P. W. Rambo, and J. Denavit, Phys. Plasmas 1, 4050 (1994).

[6] R. T. Eagleton, J. M. Foster, P. A. Rosen, and P. Graham, Rev. Sci. Instrum. 68, 834 (1997).

[7] S. S. Harilal, C. V. Bindhu, and H.-J. Kunze, Journal of Applied Physics 89, 4737 (2001).

[8] P. F. Cunningham, R. Weber, P. Ladrach, and J. E. Balmer, Optics Communications 68, 412 (1988).

[9] R. A. Bosch, R. L. Berger, B. H. Failor, N. D. Delamater, and G. Charatis, Phys. Fluids B 4, 979 (1992).

[10] A. S. Wan, T. W. Barbee, R. C. Jr., P. Celliers, L. B. D. Silva, J. C. Moreno, P. W. Rambo, G. F. Stone, J. E. Trebes, and F. Weber, Physical Review E 55, 6293 (1997).

[11] B. R. Benware, C. D. Macchietto, C. H. Moreno, and J. J. Rocca, Physical Review Letters 81, 5904 (1998).

[12] J. Filevich, K. Kanizay, M. C. Marconi, J. L. A. Chilla, and J. J. Rocca, Optics Letters 25, $356(2000)$.

[13] J. Filevich, J. J. Rocca, M. C. Marconi, R. F. Smith, J. Dunn, R. Keenan, J. R. Hunter, S. J. Moon, J. Nilsen, A. Ng, and V. N. Shlyaptsev, Applied Optics 43, 3938 (2004).

[14] C. D. Macchietto, B. R. Benware, and J. J. Rocca, Optics Letters 24, 1115 (1999).

[15] Y. Liu, M. Seminario, F. G. Tomasel, C. Chang, J. J. Rocca, and D. T. Attwood, Physical Review A 63, 033802 (2001). 
[16] J. Filevich, J. J. Rocca, M. C. Marconi, S. J. Moon, J. Nilsen, J. H. Scofield, J. Dunn, R. F. Smith, R. Keenan, J. R. Hunter, and V. N. Shlyaptsev, Physical Review Letters 94, 035005 (2005).

[17] J. Filevich, J. Grava, M. Purvis, M. C. Marconi, J. J. Rocca, J. Nilsen, J. Dunn, and W. R. Johnson, Physical Review E (Statistical, Nonlinear, and Soft Matter Physics) 74, 016404 (2006).

[18] J. Filevich, J. Grava, M. Purvis, M. C. Marconi, J. J. Rocca, J. Nilsen, J. Dunn, and W. R. Johnson, Laser and Particle Beams 25, 1 (2007).

[19] M. M. Marinak, G. D. Kerbel, N. A. Gentile, O. Jones, D. Munruo, S. Pollaine, T. R. Dittrich, and S. W. Haan, Physics of Plasmas 8, 2275 (2001).

[20] M. M. Marinak, S. W. Haan, T. R. Dittrich, R. E. Tipton, and G. B. Zimmerman, Phys. Plasmas 5 (1998).

[21] Y. T. Lee, and R. M. More, Phys. Fluids 27, 1273 (1984).

[22] M. E. Jones, D. S. Lemons, R. J. Mason, V. A. Thomas, and D. Winske, Journal of Computational Physics 123, 169 (1996). 


\section{Figures}

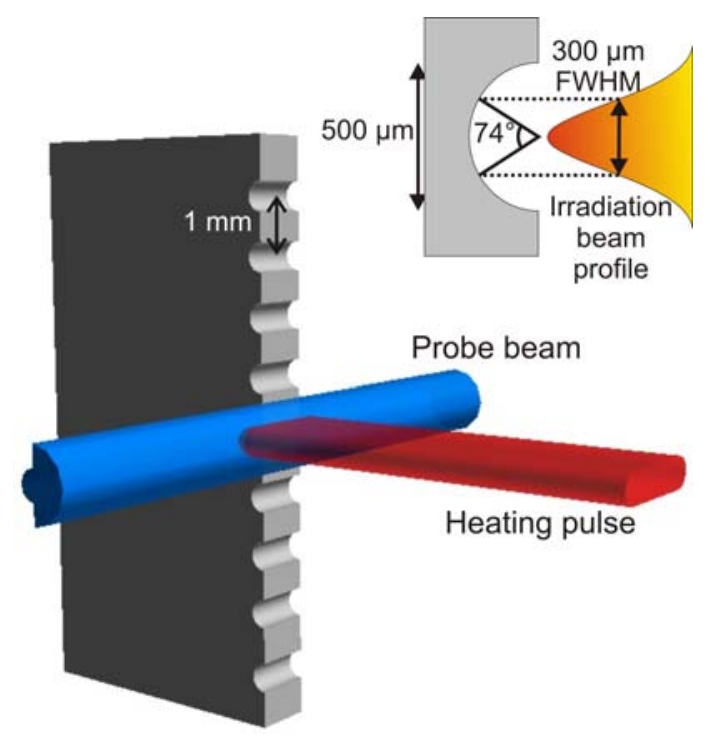

FIG. 1. 3D representation of the target with the (blue) $\lambda=46.9 \mathrm{~nm}$ probe lasers path and the (red) $\lambda=800 \mathrm{~nm}$, heating lasers path. The inset figure illustrates the $300 \mu \mathrm{m}$ FWHM Gaussian profile of the heating beam impinging onto the $500 \mu \mathrm{m}$ diameter cavity. The FWHM of the beam, which contains $77 \%$ of the energy, heats the target surface contained within an angle of 74 degrees. 


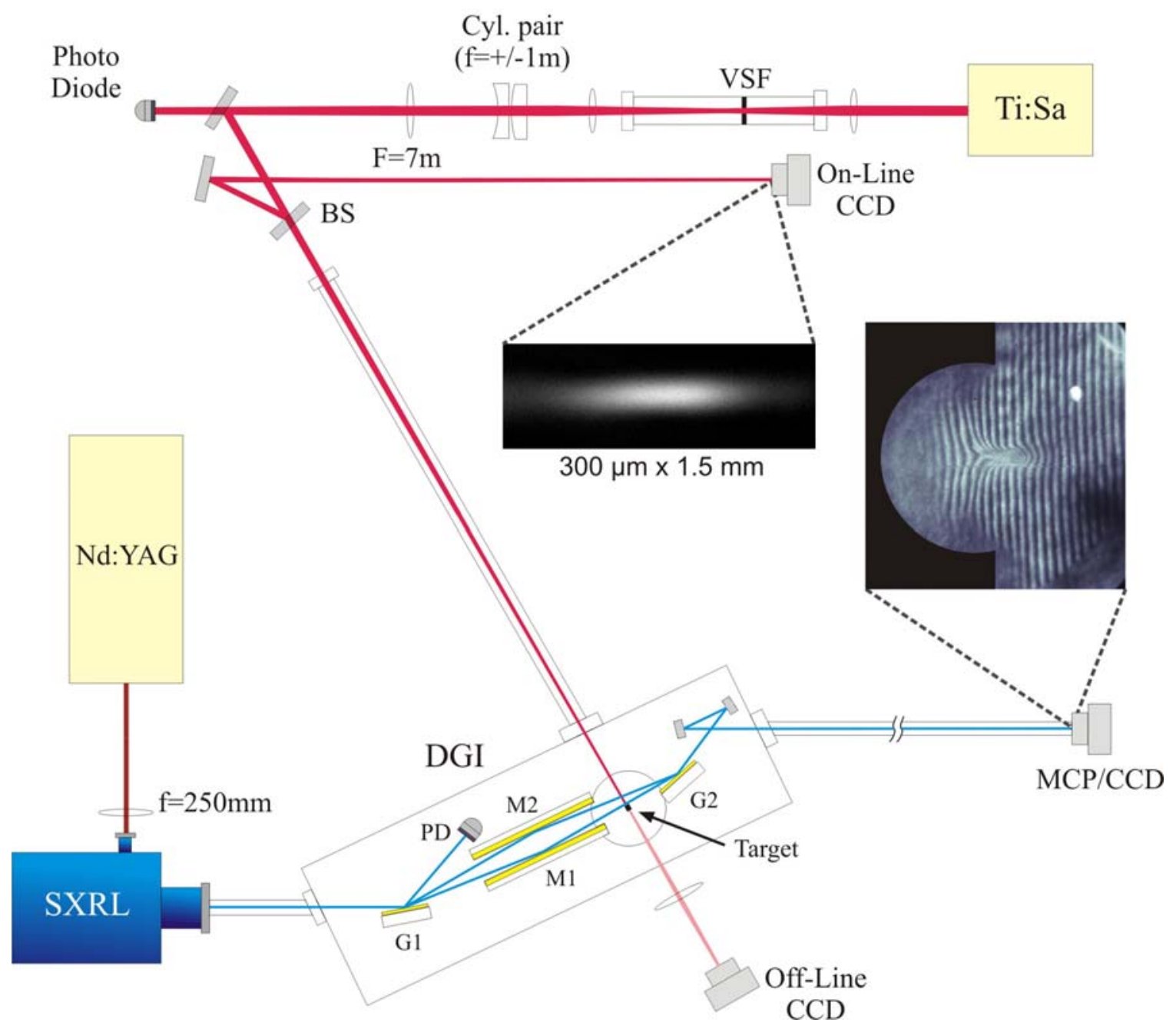

FIG. 2. Schematic of the soft $x$-ray laser interferometer system including the Ti:Sapphire heating laser and focusing optics. 

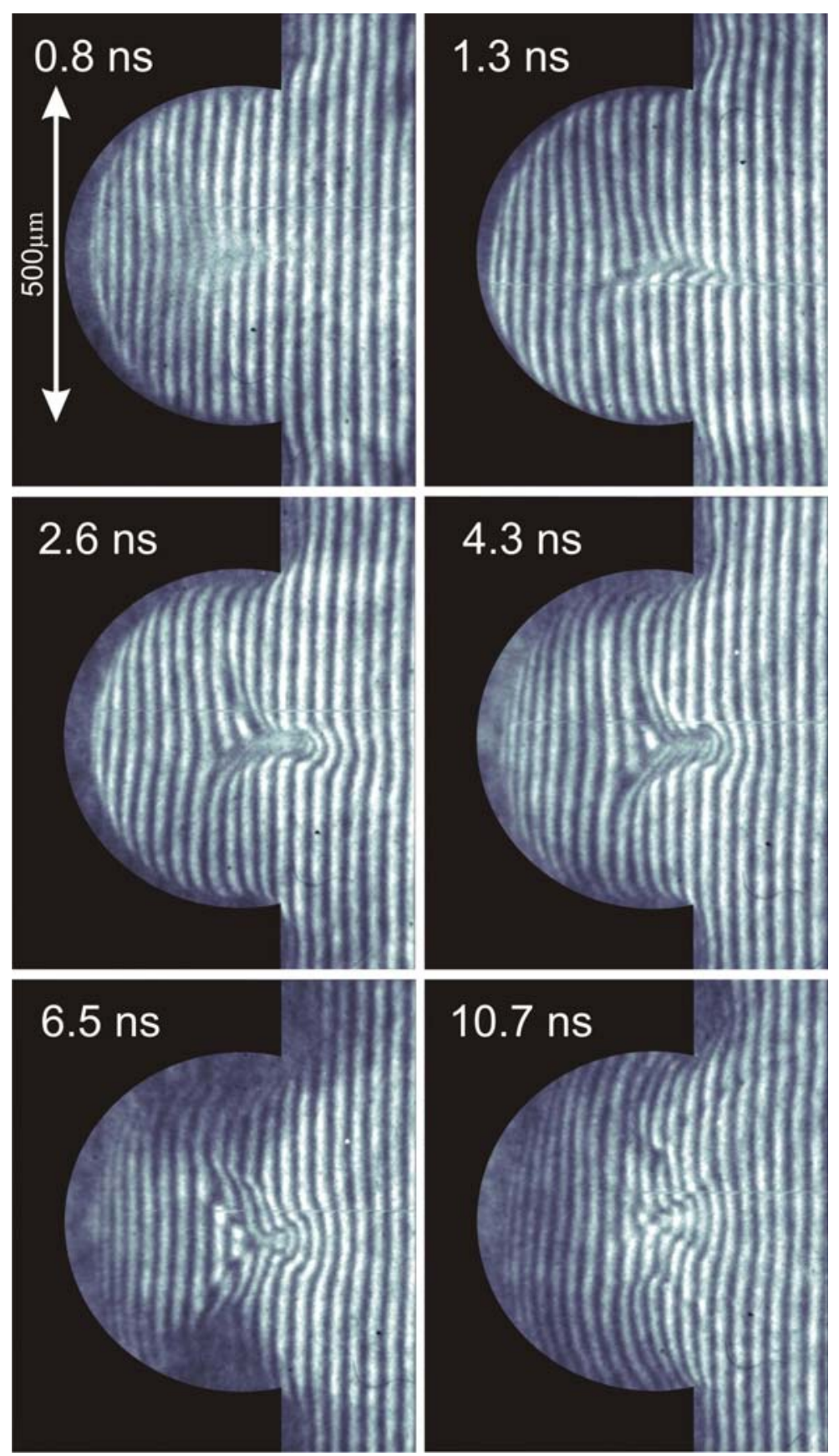

FIG. 3. Sequence of soft x-ray laser interferograms describing the evolution of plasmas created by irradiating a semi-cylindrical Al cavity with a 120 ps pulse at an intensity of $1.1 \times 10^{12} \mathrm{Wcm}^{-}$ ${ }^{2}$. Time delays are measured with respect to the peak of the irradiation laser beam incident from the right. The large number of fringe shifts close to the axis of the groove is indicative of a high density region created by the converging plasma. 


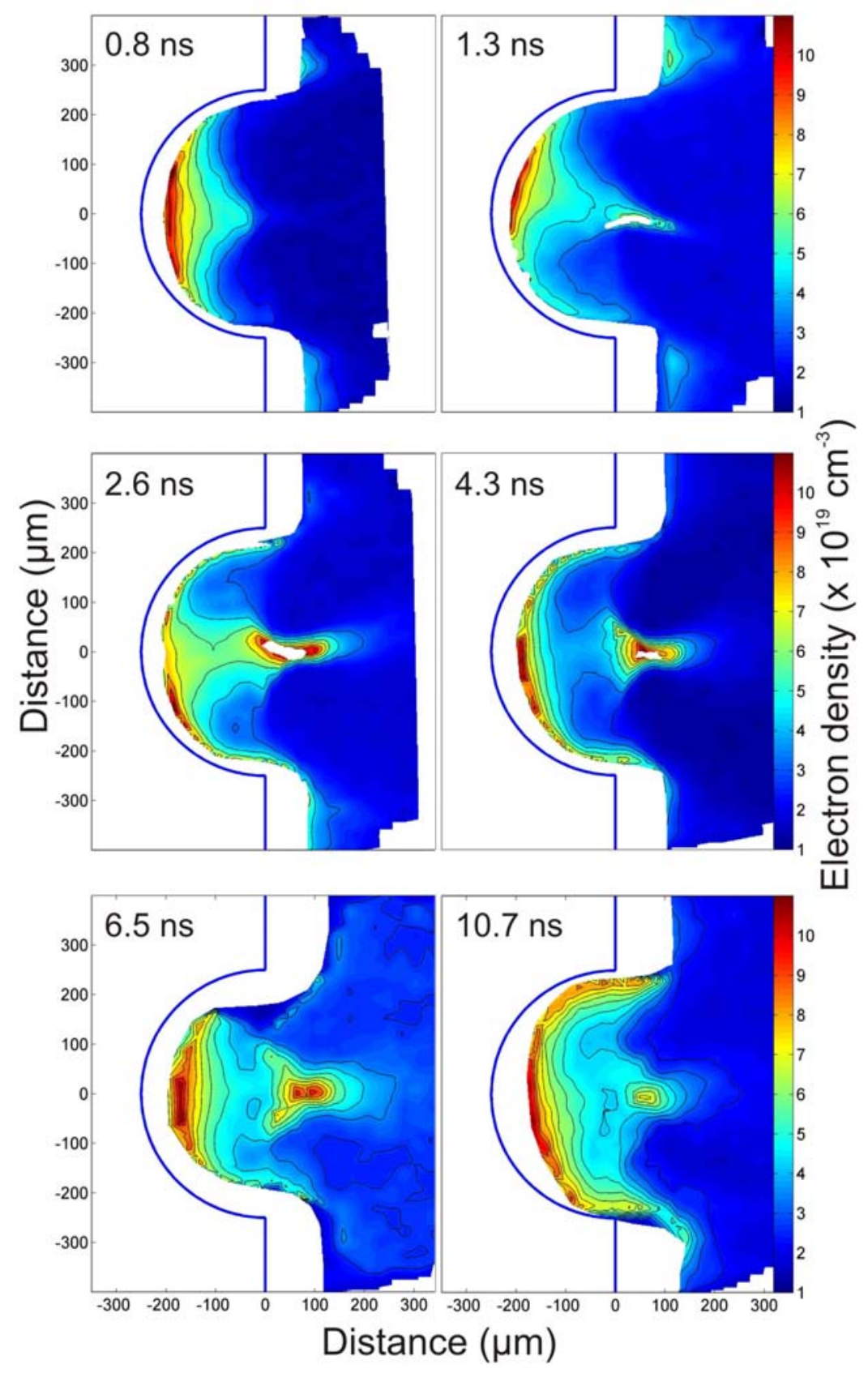

FIG. 4. Measured electron density maps of the aluminium plasma corresponding to the interferograms of Fig. 3. 


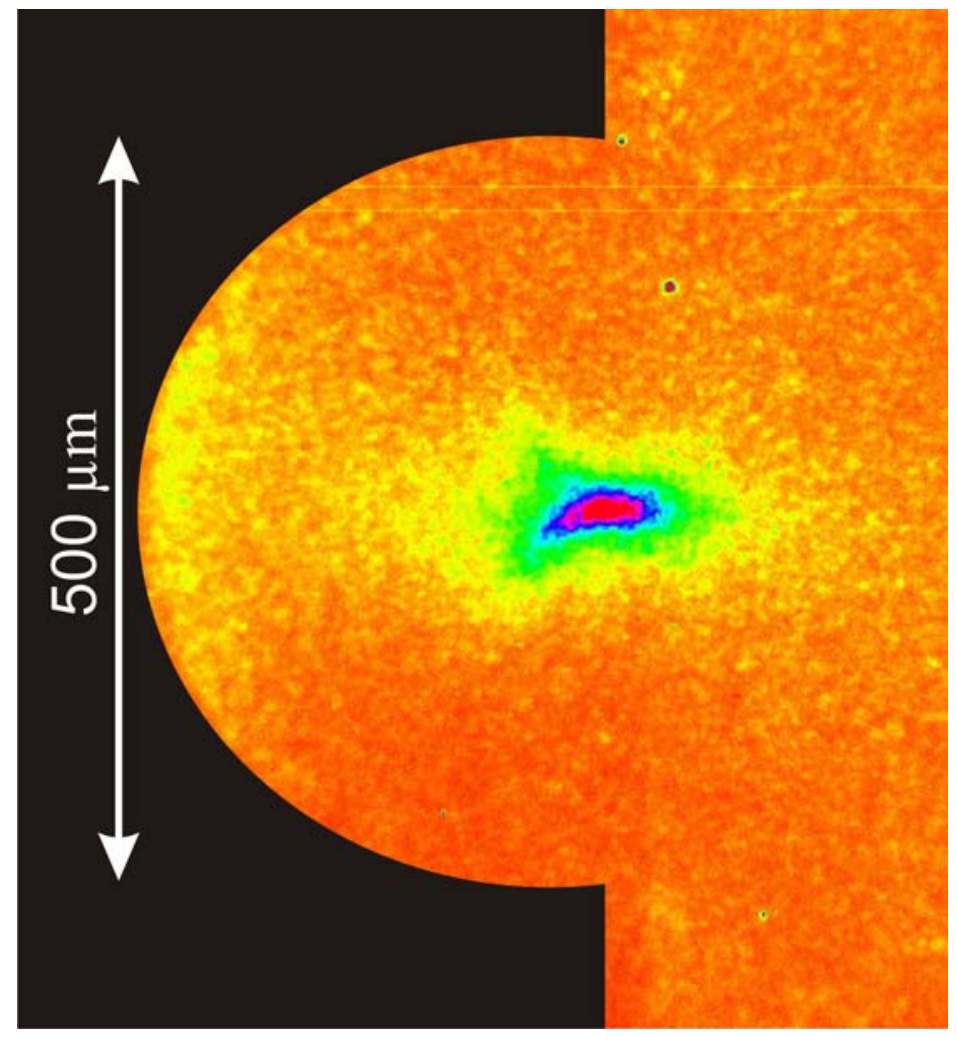

FIG. 5. Integrated self emission from the aluminum plasma. 


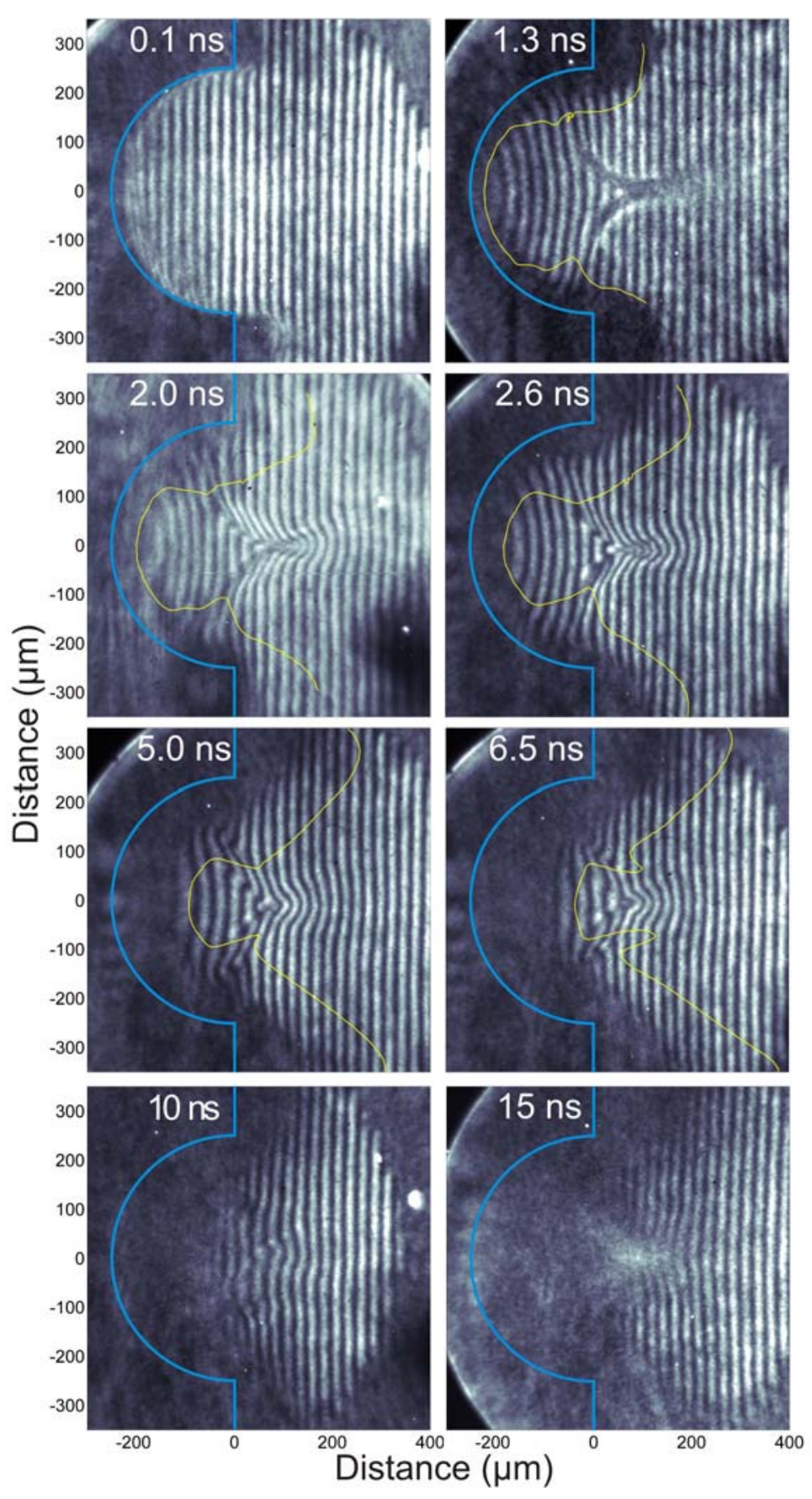

FIG. 6. Sequence of soft x-ray laser interferograms describing the evolution of carbon plasmas created under the same irradiation conditions as the aluminum interferograms in Fig. 3. The free electron approximation to the index of refraction is computed to be valid in the region to the right of the white line. 


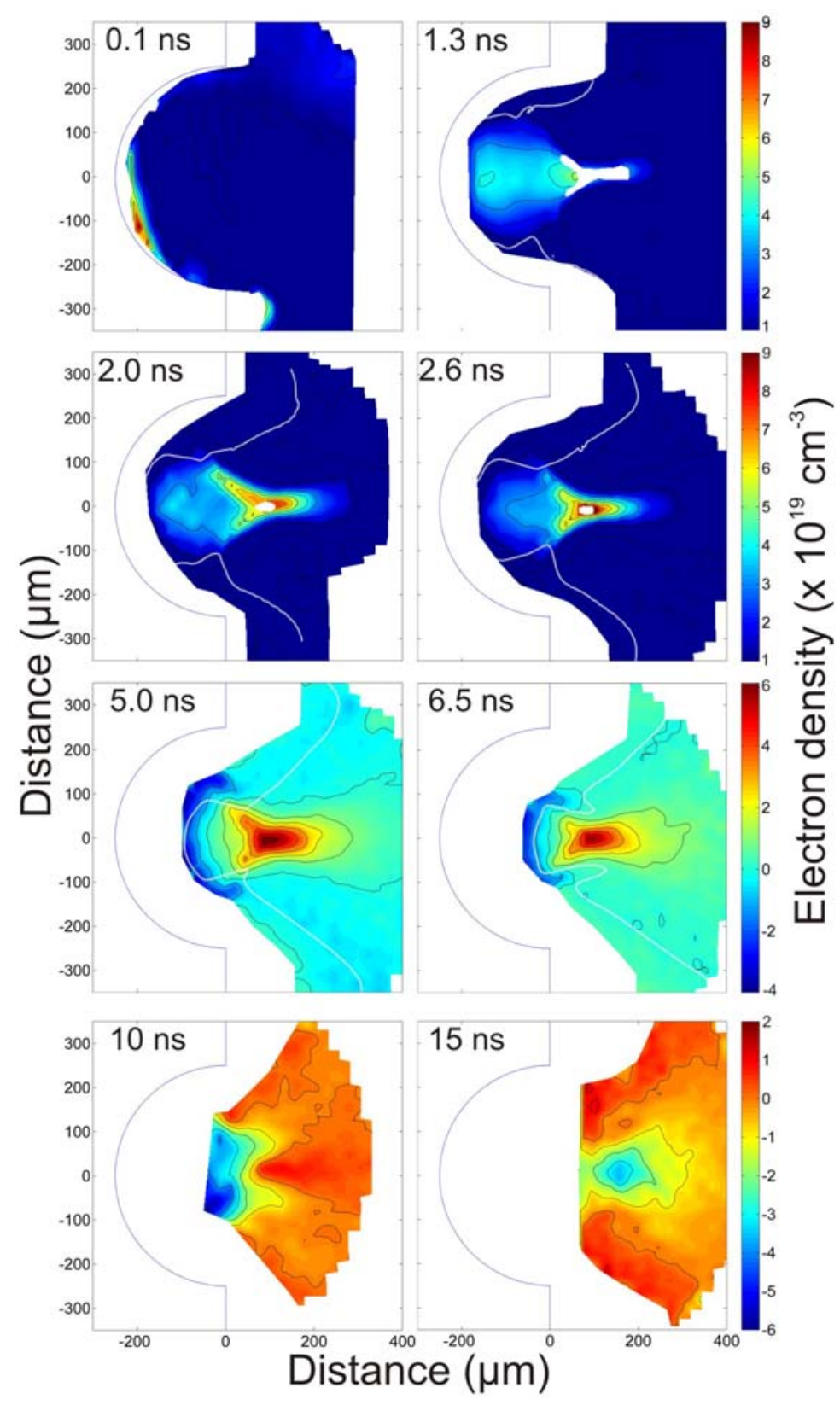

FIG. 7. Measured carbon electron density maps corresponding to the interferograms shown in Fig. 6. An electron density color bar is shown at the right with units of $10^{19} \mathrm{~cm}^{-3}$. The free electron approximation to the index of refraction is computed to be valid in the region to the right of the white line. 


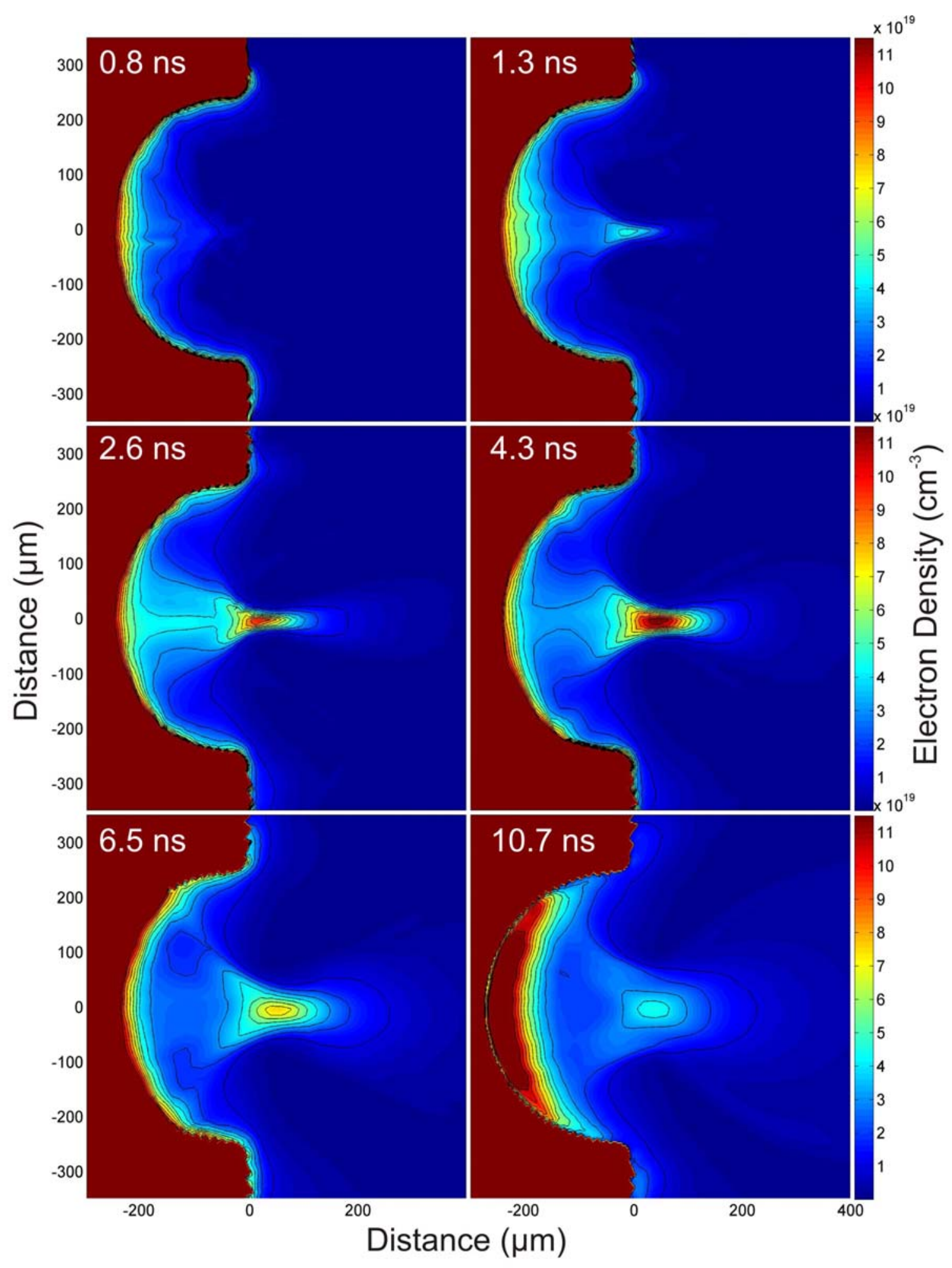

FIG. 8. Simulated electron density maps for the aluminum plasmas. 


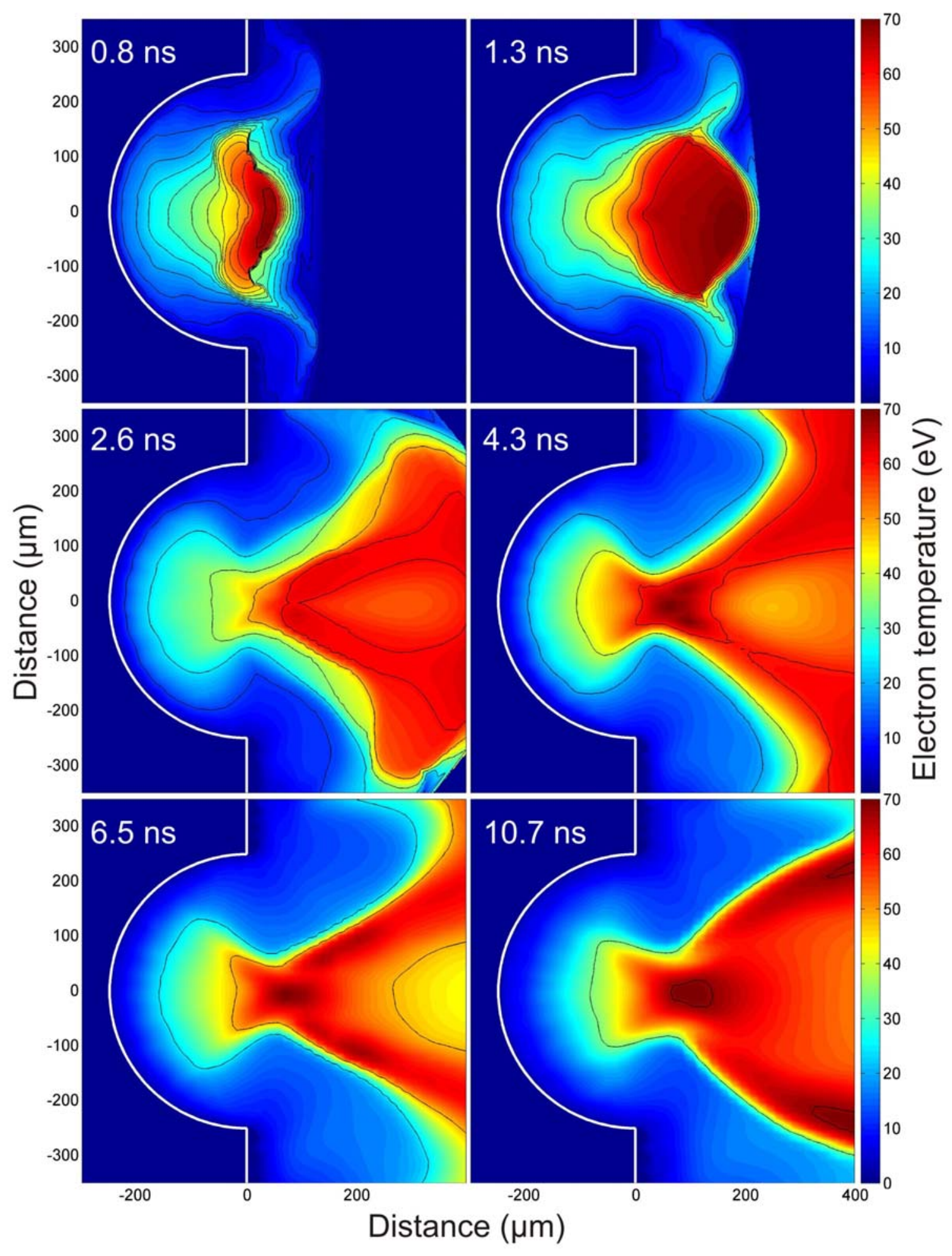

FIG. 9. Simulated electron temperature maps for the aluminum plasmas. 


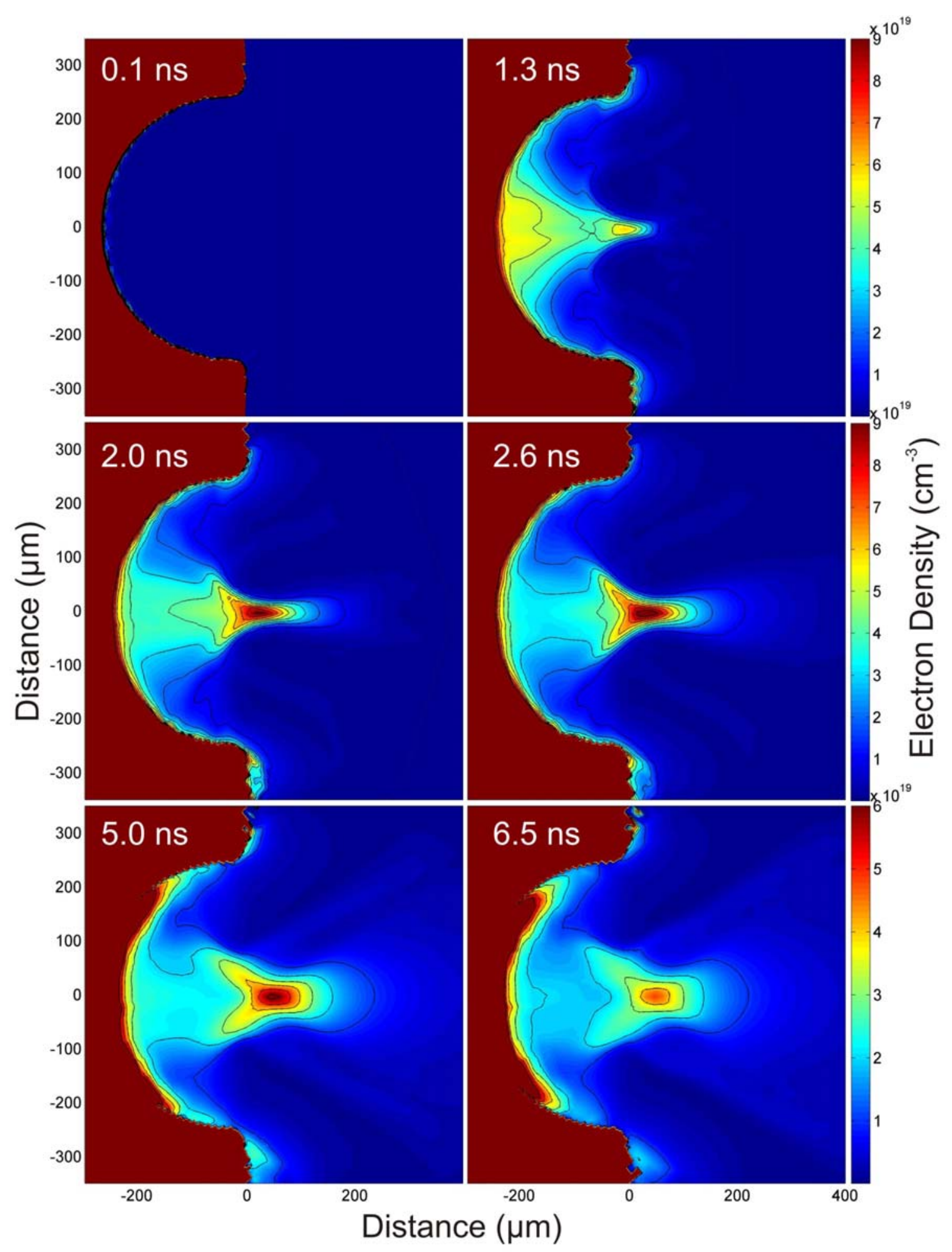

FIG. 10. Simulated electron density maps for the carbon plasmas. 


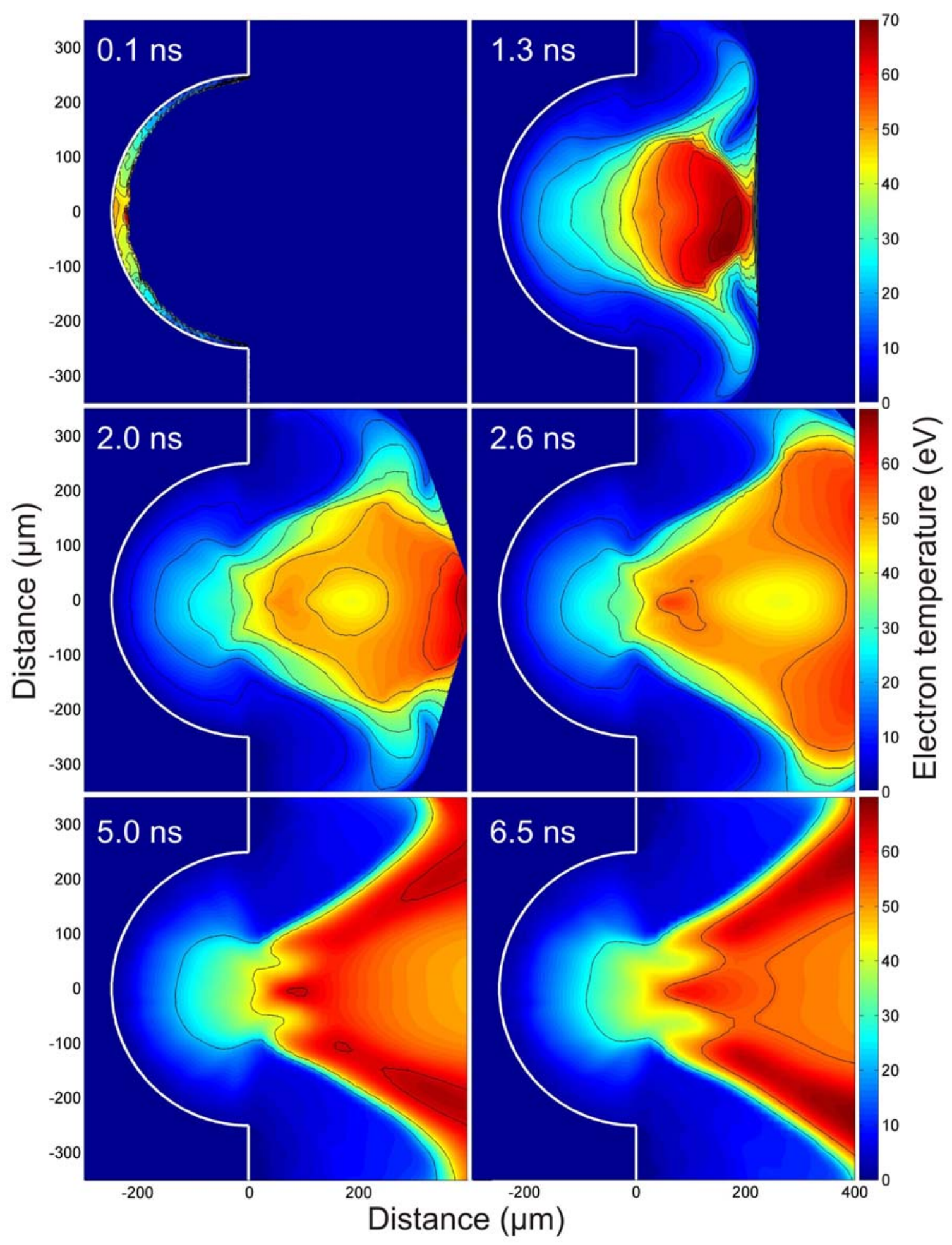

FIG. 11. Simulated electron temperature maps for the carbon plasmas. 


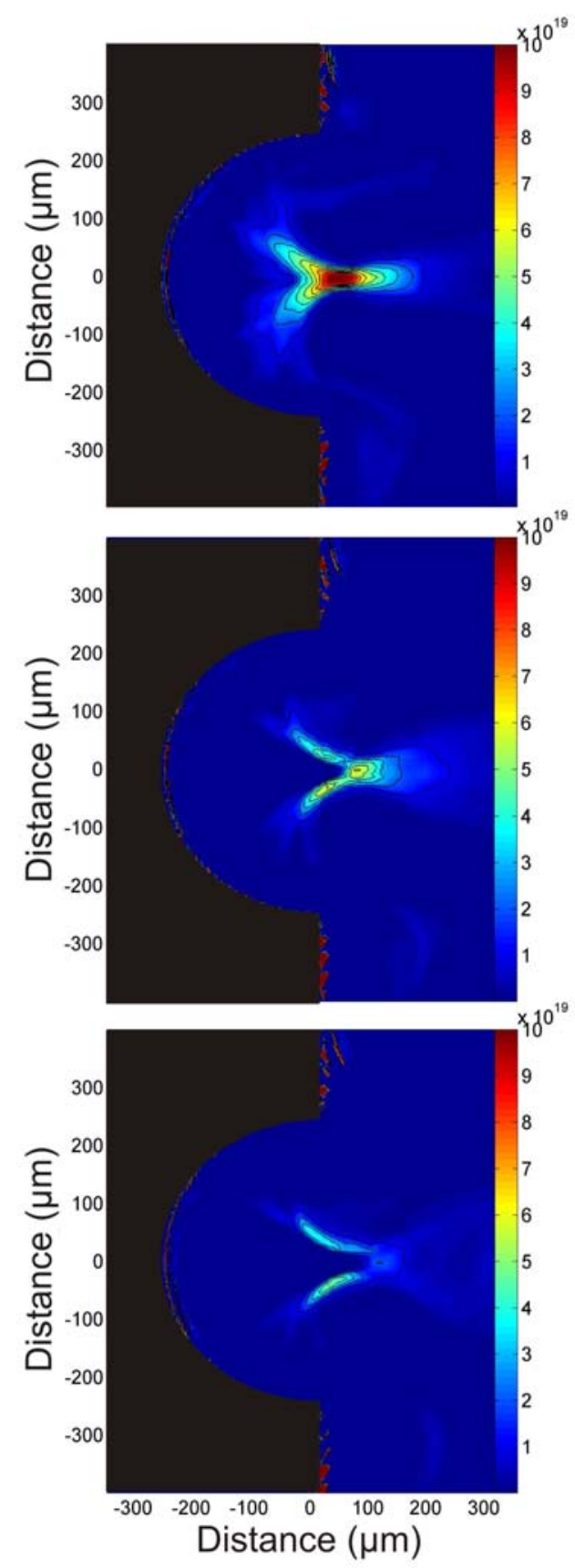

FIG. 12. Simulations showing the increase in electron density over a period of 1 ns centered at $1.3 \mathrm{~ns}, 2.0 \mathrm{~ns}$ and $2.6 \mathrm{~ns}$. A change in the electron density of $5 \times 10^{19} \mathrm{~cm}^{-3}$ corresponds to one fringe shift. Half a fringe shift causes complete blurring of the fringes in the interferograms of Fig. 6. 


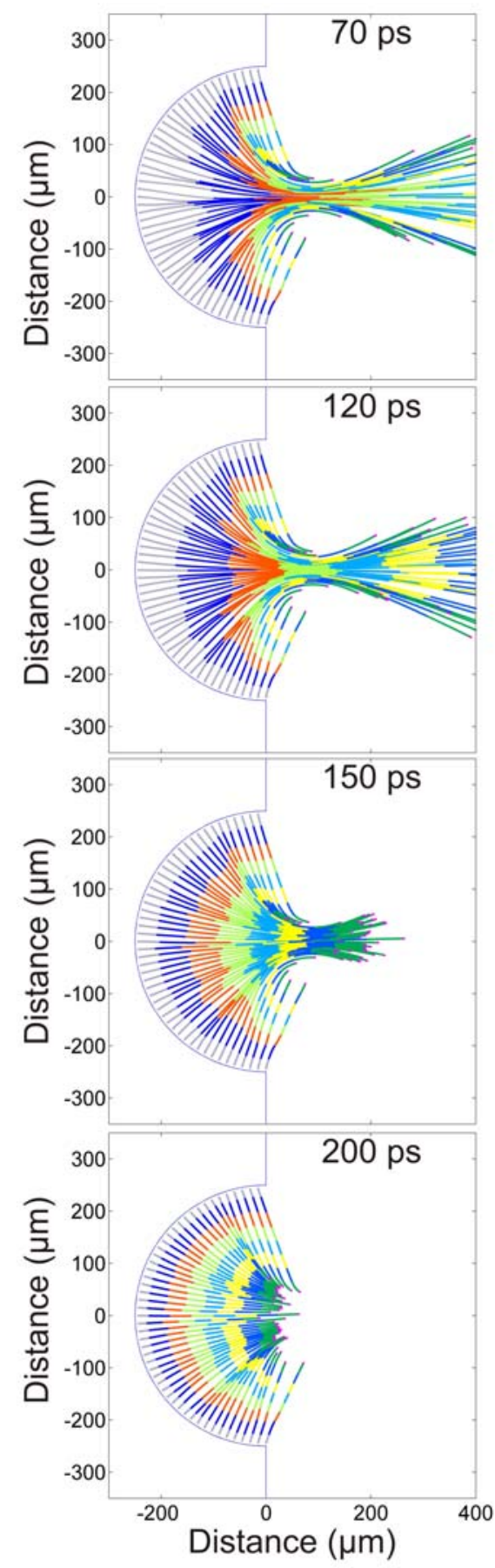

FIG. 13. Plots revealing the 4 ns trajectory evolution of tracer particles leaving the target surface at four different time delays with respect to the beginning of the laser heating pulse. Alternating colors are used to represent time periods of 500ps. 